\title{
A Model for RF Loss through Vegetation with Varying Water Content
}

\author{
Sonam Peden ${ }^{*}$, Ronald C. Bradbury ${ }^{1}$, David William Lamb ${ }^{1,2}$, Mark Hedley ${ }^{3}$ \\ ${ }^{1}$ Precision Agriculture Research Group, University of New England (UNE), Armidale, NSW, Australia \\ ${ }^{2}$ Food Agility Cooperative Research Centre, University of New England, Armidale, NSW, Australia \\ ${ }^{3}$ CSIRO Data61, Marsfield, NSW, Australia \\ Email: *speden@myune.edu.au
}

How to cite this paper: Peden, S., Bradbury, R.C., Lamb, D.W. and Hedley, M. (2021) A Model for RF Loss through Vegetation with Varying Water Content. Journal of Electromagnetic Analysis and Applications, 13, 41-56.

https://doi.org/10.4236/jemaa.2021.133003

Received: March 11, 2021

Accepted: March 28, 2021

Published: March 31, 2021

Copyright $\odot 2021$ by author(s) and Scientific Research Publishing Inc. This work is licensed under the Creative Commons Attribution International License (CC BY 4.0).

http://creativecommons.org/licenses/by/4.0/

\begin{abstract}
Assessing plant water status is important for monitoring plant physiology. Radio signals are attenuated when passing through vegetation. Both analytical and empirical models developed for radio frequency (RF) loss through vegetation have been dependent on experimental measurements and those measurements have been completed in specific situations. However, for models to be more broadly applicable across a broad range of vegetation types and constructs, basic electrical properties of the vegetation need to be characterised. Radio waves are affected especially by water and the relationship between water content in vegetation expressed as effective water path (EWP) in $\mathrm{mm}$ and measured RF loss (dB) at $2.4 \mathrm{GHz}$ was investigated in this work. The EWP of eucalyptus leaves of varying amounts of leaf moisture $(0 \%-41.5 \%)$ ranged from $0-14 \mathrm{~mm}$, respectively. When the model was compared with the actual RF loss there was a systematic offset equivalent to a residual leaf moisture content of $6.5 \%$ that was unaccounted for in the leaf moisture content determination (oven drying). This was attributed to bound water. When the model was adjusted for this amount of additional leaf water, the average RMSE in predicted RF loss was $\pm 2.2 \mathrm{~dB}$ and was found to explain $89 \%$ of the variance in measured RF loss.
\end{abstract}

\section{Keywords}

Radio Attenuation, Leaves, Water Content, Path Loss, Vegetation Thickness, Permittivity

\section{Introduction}

Radio signals are attenuated when passing through vegetation due to absorption and scattering by the discrete elements such as the branches, stems and leaves 
[1] [2] [3]. The so-called RF loss has been measured for specific frequencies in particular situations (forest, apple orchard \& coconut garden for example) and empirical models have been developed from such measurements [2] [4]-[8]. Analytical models have also been developed but the more accurate Radiative Energy Transfer (RET) models depend on experimental measurements for their formulation and validation [9]. For a model to be accurate across a broad range of vegetation however, the relevant electrical characteristics of the vegetation need to be incorporated into the model.

The detection of plant water status is important for monitoring the physiological status of plants, the assessment of drought and fire risk in natural plant communities, and for irrigation scheduling of crops [10] [11]. Although field sampling of single leaves and shoots provides the most accurate assessment of plant water status, such methods are not feasible when estimates are required for large areas of vegetation [12].

Radio waves interact strongly with water [13] and eucalyptus leaves are no exception. While RF loss measurements at any radio frequency would be related to water content, some frequency bands are more suitable than others. Below about $600 \mathrm{MHz}$, the main RF loss mechanism involves the movement of ions. RF loss then is highly dependent on the medium's electrical conductivity and hence the concentration of dissolved ions. Such information would be difficult to obtain for different species of vegetation which presents a hurdle for practical estimation of water content. Above about $1 \mathrm{GHz}$ and up to $100 \mathrm{GHz}$, the main RF loss mechanism is the rotation of water molecules resulting from the interaction between the radio signal electric field and the molecular electric dipole [14] [15]. Frequencies above $1 \mathrm{GHz}$ have an advantage in that electrical conductivity does not play a significant part hence avoiding the need to characterise the highly-variable constituents of electric conductivity in leaves. The higher frequency also offers another advantage; namely directional antennas that can be used to facilitate location-specific measurements are smaller.

Le Vine and Karam [16] calculated the attenuation associated with a vegetation canopy using a discrete scatter model, where the vegetation canopy is presented by a sparse layer of discrete, randomly oriented particles such as leaves, stalks, branches, etc. over a homogeneous ground plane (soil). They found that for frequencies up to $5 \mathrm{GHz}$ the attenuation varies linearly with plant water content but for optical frequencies, the attenuation is relatively independent of both water content and frequency. Nakajima, Ohyama, Juzoji and Ta [17] measured the RF attenuation of individual leaves at 5, 10 and $20 \mathrm{GHz}$ in a waveguide. They also investigated the effect in a living tree by measuring RF attenuation at 10.5 GHz. They asserted that "Microwave attenuation by tree foliage should have a strong link to water content in the leaves". The dependency of attenuation on water content is through the dielectric constant which is highly dependent on the water content inside the material. Furthermore, moisture on the surface of leaves of the trees also absorbs the RF waves resulting in more attenuation [18] [19] [20] [21]. 
The water inside leaves/vegetation can be divided into free water and bound water. Free water is the liquid water found in cell lumen and is relatively easy to remove [22]. Bound water is the water molecules that penetrate the cell walls and are chemically bound to cellulose molecules. Bound water cannot always be expelled by heat without damaging the material [23] and the removal of bound water also depends on the temperature and relative humidity of the environment [24].

The aim of this paper is to extend the model of complex permittivity for vegetation developed by Ulaby and El-Rayes [25] with the view to developing a means of estimating water content of vegetation. They developed a Debye-Cole dual-dispersion model for complex relative permittivity (also known as complex dielectric constant) consisting of three parts: a dispersive free-water component, a dispersive bound water component and a nondispersive residual component. Their proposed dielectric model was found to give excellent agreement with data over a wide range of moisture conditions and over the entire $0.2-20 \mathrm{GHz}$ range examined in their study. In this paper a model for calculating the radio frequency (RF) signal loss in vegetation is derived. The model in this study uses the complex permittivity for vegetation modelled by Ulaby and El-Rayes. The model is compared against experimental measurements of RF loss for eucalyptus leaves at $2.4 \mathrm{GHz}$.

\section{Model}

\subsection{Wave Propagation}

We assume that a plane wave travels through a lossy homogeneous medium of thickness $d$ with complex relative permittivity, $\varepsilon_{V}$ as shown in Figure 1. Also, we assume that the material is non-magnetic.

Complex permittivity, $\mathcal{E}_{V}$ is expressed as

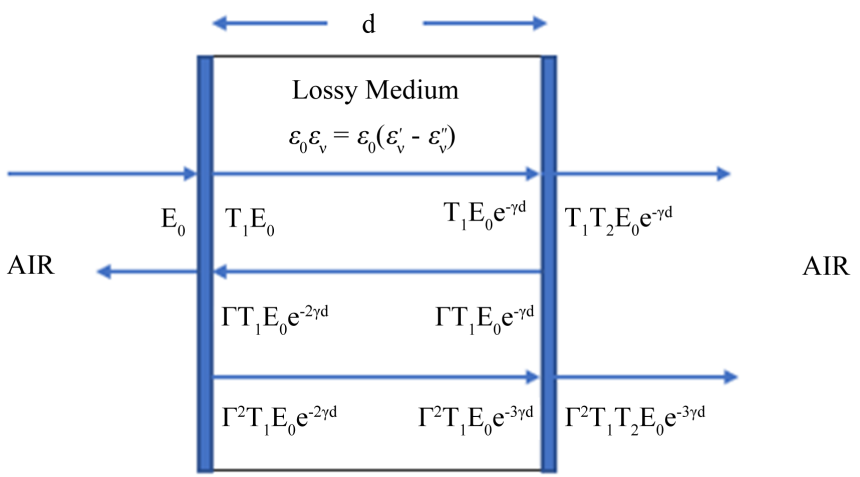

Figure 1. The medium is modelled as a parallel-sided slab of thickness $d$ with air on either side. When a plane radio wave travelling left-to-right meets the medium it is partially transmitted and partially reflected at the first interface. The transmitted wave propagates through the material with a complex propagation constant. At the second interface it is again partially transmitted and partially reflected. The total outgoing wave is a composite of the unreflected "straight through" wave and waves that have been reflected internally $2,4, \ldots$, times. 


$$
\varepsilon_{v}=\varepsilon_{v}^{\prime}-j \varepsilon_{v}^{\prime \prime}
$$

where, the real part, $\varepsilon_{v}^{\prime}$ represents the relative permittivity and the imaginary part, $\varepsilon_{v}^{\prime \prime}$ represents the dielectric loss [23].

When an incident electromagnetic wave with electric field phasor, $E_{0}$ passes through a lossy homogeneous medium, the total transmitted field phasor will be a combination of transmitted power across the interfaces and reflections from the interfaces, as shown in Figure 1.

In Figure 1, $T_{1}$ is the transmission coefficient of the wave transmitting through air-to-lossy medium interface, $T_{2}$ is the transmission coefficient of the wave transmitting through lossy medium-to-air interface, $\gamma$ is the complex propagation constant in the medium and $\Gamma$ is the reflection coefficient of the wave undergoing multiple internal (partial) reflections inside the slab at the lossy medium-to-air interfaces. These terms are expressed in the following Equations (2)-(5) [26]:

$$
\begin{gathered}
T_{1}=\frac{2}{\sqrt{\varepsilon_{v}}+1}, \\
T_{2}=\frac{2 \sqrt{\varepsilon_{v}}}{\sqrt{\varepsilon_{v}}+1}, \\
\Gamma=\frac{\sqrt{\varepsilon_{v}}-1}{\sqrt{\varepsilon_{v}}+1}, \\
\gamma=j \omega \sqrt{\mu_{0} \varepsilon_{0} \varepsilon_{v}}
\end{gathered}
$$

where, $\omega$ is the angular frequency in $\mathrm{rad} / \mathrm{sec}, \mu_{0}$ and $\varepsilon_{0}$ are the permeability and permittivity of air respectively.

The total transmitted complex electric phasor, $E$ after passing through a lossy medium slab is given by the "straight through" wave and a series of multiple-reflected waves (refer Figure 1). Depending on the relative phase shifts in the different paths, these contributions to the outgoing wave may reinforce or cancel each other. Summing these terms as phasors accounts for the phase shift

$$
E=T_{1} T_{2} \mathrm{e}^{-\gamma d} E_{0}\left(1+\Gamma^{2} \mathrm{e}^{-2 \gamma d}+\Gamma^{4} \mathrm{e}^{-4 \gamma d}+\cdots\right) .
$$

Using geometric series for $|\Gamma|<1, E$ can be expressed as

$$
E=\frac{T_{1} T_{2} E_{0} \mathrm{e}^{-\gamma d}}{1-\Gamma^{2} \mathrm{e}^{-2 \gamma d}}
$$

The total loss for the lossy homogenous slab in $\mathrm{dB}$ is

$$
L_{\text {slab }}=20 \log _{10}\left|\frac{E_{0}}{E}\right| \text {. }
$$

Replacing Equations (2)-(4) \& (7) in Equation (8) yields

$$
L_{\text {slab }}=20 \log _{10}\left|\left(\left(\sqrt{\varepsilon_{v}}+1\right)^{2}-\left(\sqrt{\varepsilon_{v}}-1\right)^{2}\left(\mathrm{e}^{-2 \gamma d}\right)\right) /\left(4 \sqrt{\varepsilon_{v}} \mathrm{e}^{-\gamma d}\right)\right| \text {. }
$$




\subsection{Complex Permittivity of Vegetation}

If the lossy homogenous medium is leaves, then the permittivity $\varepsilon_{V}$ in Equation (9) is the permittivity of vegetation. Ulaby and El-Rayes [25] developed a dielectric model to calculate the dielectric constant of vegetation. They modelled the dielectric constant of vegetation, $\varepsilon_{v}$ as a simple addition of three components: a nondispersive residual component $\left(\varepsilon_{I}\right)$, free-water component $\left(v_{f w} \varepsilon_{f}\right)$ and the bulk vegetation-bound water component $\left(v_{b} \varepsilon_{b}\right)$, expressed as

$$
\varepsilon_{v}=\varepsilon_{r}+v_{f w} \varepsilon_{f}+v_{b} \varepsilon_{b}
$$

where, $V_{f w}$ is the volume fraction of free water, $\varepsilon_{f}$ is the dielectric constant of free water, $v_{b}$ is the volume fraction of the bulk vegetation-bound water mixture and $\varepsilon_{b}$ is its dielectric constant. Assuming that $\varepsilon_{r}$ is a nondispersive residual component is supported by Hasted [23] who states that the dielectric loss of many dry materials is low in the microwave band, having values between $10^{-1}$ and $10^{-3}$.

Free water may contain dissolved salt and the frequency dependent dielectric constant of bulk saline water is given by the Debye equation [27],

$$
\varepsilon_{f}=\varepsilon_{f}^{\prime}-j \varepsilon_{f}^{\prime \prime}=\varepsilon_{f \infty}+\frac{\left(\varepsilon_{f s}-\varepsilon_{f \infty}\right)}{1+j\left(f / f_{f 0}\right)}-j \frac{\sigma}{2 \pi f \varepsilon_{0}}
$$

where, $f$ is the operating frequency in $\mathrm{Hz}, f_{00}$ is the relaxation frequency in $\mathrm{Hz}$, and $\varepsilon_{f s}$ and $\varepsilon_{f \infty}$ are the dimensionless static and high frequency limits of $\varepsilon_{f}^{\prime}$. The salinity, $S$ of a solution is defined as the total mass of salt in grams dissolved in a solution of $1 \mathrm{~kg}$ and is expressed as parts per thousand on a weight basis. The salinity for vegetation is taken to be $10 \%$ [28]. For salinity, $S \leq 10 \%$ and at room temperature, Equation (11) could be approximated as,

$$
\varepsilon_{f}=4.9+\frac{75}{1+j(f / 18)}-j \frac{\sigma 18}{f}
$$

where, $f$ is in $\mathrm{GHz}$.

The conductivity $\sigma$ (siemen/metre) may be related to $S(\%$ ) by,

$$
\sigma \cong 0.16 S-0.0013 S^{2} \text {. }
$$

For bound water, Ulaby and El-Rayes [25] conducted dielectric measurements on sucrose-water mixture and data was fitted to Cole-Cole dispersion equation. The complex dielectric constant of bound water is given by

$$
\varepsilon_{b}=2.9+\frac{55}{1+(j f / 0.18)^{0.5}}
$$

where, $f$ is in GHz. Equation (11) includes a loss term associated with the conductivity of the free water and dissolved ions in the medium. In contrast, Equation (14) has no corresponding conductivity term as the water molecules are bound to other substances and do not contribute to the bulk conductivity of the medium.

By inserting Equations (12)-(14) in Equation (10), the dielectric constant of vegetation can be written as 


$$
\varepsilon_{v}=\varepsilon_{r}+v_{f w}\left(4.9+\frac{75}{1+j(f / 18)}-j \frac{\sigma 18}{f}\right)+v_{b}\left(2.9+\frac{55}{1+(j f / 0.18)^{0.5}}\right)
$$

The variation of $\varepsilon_{r}, V_{f w}$ and $v_{b}$ with gravimetric moisture content, $M_{g}$ were derived by Ulaby and El-Rayes [25] by fitting their model (Equation (15)) to complex permittivity measurements acquired using corn leaves and verified against corn stalks, soybean leaves, aspen leaves, balsam fir trunk, potatoes, apples, and other types of vegetation material. The empirical equations are as follows:

$$
\begin{gathered}
\varepsilon_{r}=1.7-0.74 M_{g}+6.16 M_{g}^{2}, \\
v_{f w}=M_{g}\left(0.55 M_{g}-0.076\right), \\
v_{b}=\left(4.64 M_{g}^{2}\right) /\left(1+7.36 M_{g}^{2}\right),
\end{gathered}
$$

where, $M_{g}$ is calculated from the weight measurement of leaves before and after drying them inside a vacuum oven as follows

$$
M_{g}=1-\left(\frac{\text { weight of dry leaves }}{\text { weight of leaves }(\text { different stages of drying })}\right) \text {. }
$$

\section{Material and Method}

All the experiments were carried out at The University of New England main campus located in Armidale, New South Wales, Australia (latitude $30.4867^{\circ} \mathrm{S}$ and longitude $151.6430^{\circ} \mathrm{E}$ ). Two flat-panel, phased-array directional antennas (ARC Wireless Solutions, USA, PA2419B01, $39.1 \mathrm{~cm} \times 39.1 \mathrm{~cm} \times 4.3 \mathrm{~cm}$ ) were used, one as a transmitter connected to a transceiver Beacon (Dosec Design, Australia, EnviroNode Beacon) and the other as a receiver connected to a transceiver hub (Dosec Design, Australia, EnviroNode Hub) operated at a frequency of $2.4331 \mathrm{GHz}$. The antenna had a gain of $19 \mathrm{dBi}$, front-to-back ratio of $>30 \mathrm{~dB}$ and $3 \mathrm{~dB}$ beamwidth of $\pm 9^{\circ}$. The antennas were placed outside, facing each other at a separation of 6.10 metres. A constant transmitted power of 100 milliwatts was used. The hub measured and logged the RSSI (received signal strength indicator, $\mathrm{dBm}$ ) to a removable $\mathrm{SD}$ card at 1 minute intervals. The experimental set-up is shown in Figure 2.

Some preliminary measurements were made to verify the experimental set-up. A wooden frame covered, front and back, with $5 \mathrm{~mm}$ clear acrylic sheets was used to hold the leaves to be tested. In order to confirm that only the radio waves propagating directly through the wooden frame were received by the receiver (i.e. no multipath signals), a metal sheet, impenetrable to radio waves, was temporarily attached to the front of the frame. The position of the frame relative to the transceivers was then optimised such that the RSSI was below the minimum measurable power level for our equipment $(-80 \mathrm{dBm})$.

In order to measure any potential effects of the wooden frame itself, each of the empty frames was positioned between the transceivers and the RF signal loss, relative to no frame in place, was measured. Irrespective of frame size, the RF 


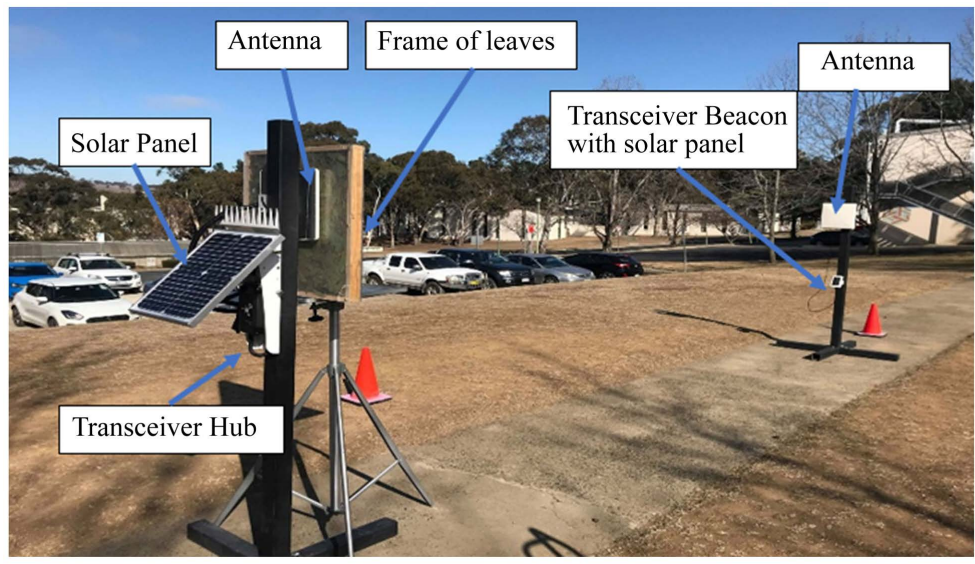

Figure 2. Experimental set-up. Two flat-panel antennas were mounted facing each other $6.10 \mathrm{~m}$ apart. A frame containing the leaves being tested was mounted on a tripod immediately in front of one antenna. Solar panels for electrical power are also visible. The RSSI $(\mathrm{dBm})$ was recorded to a removable SD card inside the transceiver Hub every minute.

loss through the empty frame was confirmed to be below the measurement resolution of $0.1 \mathrm{~dB}$, which indicated that any loss associated with the frame of leaves would be solely due to the presence of the contained material inside.

A wooden frame (inner dimensions $600 \mathrm{~mm} \times 560 \mathrm{~mm} \times 42 \mathrm{~mm}$ ) was completely filled with freshly-plucked, turgid Eucalyptus laevopinea (silver top stringybark) leaves such that there was no visible gap between the frame and leaves when the frame is flipped over. The filled frame was then weighed. The RSSI $(\mathrm{dBm})$ for no obstruction between the transceivers was measured for 3 minutes and then the frame filled with leaves was placed on the stand and signal strength was again measured for 3 minutes. The difference between the time-average RSSI with and without frame in place was converted to a time-averaged RF loss associated with the leaves. The sequence of frame and no frame measurements was repeated three times to provide a measurement average. The RF loss $(L)$ associated with the sample of leaves was then calculated using,

$$
L(\mathrm{~dB})=\operatorname{RSSI}(\text { no frame })-\operatorname{RSSI}(\text { frame + leaves }) .
$$

Following the RSSI measurements with and without the frame in place, the leaves were removed from the frame and oven-dried in a vacuum oven at a temperature of $60^{\circ} \mathrm{C}$ and $80 \mathrm{kPa}(60 \mathrm{cmHg})$ vacuum gauge pressure for one hour. As condensation and the temperature of the transmitting material effects radio wave transmission, the hot leaves were then spread on a table surface to both cool down and allow residual water vapour to dissipate rather than condense. The frame was then refilled with the cooled leaves and weighed. The frame containing the now partially-dried leaves was then placed on the stand at the experimental site and the process of measuring the RSSI was repeated.

The process of partial drying and remeasuring the RSSI was repeated until no further weight loss from drying was achieved. At this point, the desiccated leaves were yellow/brown in colour and brittle. 
At this end point the mass of the water $\left(\mathrm{m}_{\mathrm{w}}\right)$ in the freshly plucked and each partially-dried, leaf sample was retrospectively calculated from the known mass of the leaf samples and the final dry weight of the desiccated leaves.

The measurement sequence was repeated for frame thicknesses of $63 \mathrm{~mm}, 105$ $\mathrm{mm}, 147 \mathrm{~mm}$ and $195 \mathrm{~mm}$. Any RF loss measurements exceeding $30 \mathrm{~dB}$, as occurred in the $147 \mathrm{~mm}$ and $195 \mathrm{~mm}$ frame thicknesses, exceeded the reliable measurement range of the equipment and were excluded from subsequent analyses.

The radio wave passes through vegetation thickness, $d_{v}$ containing a distributed mass, $\mathrm{m}_{\mathrm{w}}$ of water $(\mathrm{kg})$. In order to quantify the equivalent amount of water affecting the radio wave, we introduce the effective water path (EWP) as the thickness of a sheet of $100 \%$ water with the same mass. If the dimensions of the frame are $d_{r} x$, and $y$, then the water mass is

$$
m_{w}=\rho_{w} \times \mathrm{EWP} \times x \times y,
$$

where, $\rho_{w}$ is the density of pure water $1000 \mathrm{~kg} / \mathrm{m}^{3}$. Hence, EWP in $\mathrm{mm}$ can be expressed as

$$
\mathrm{EWP}=\left(\frac{m_{w} \times d_{v}}{\rho_{w} \times V}\right) \times 1000
$$

where, $V$ is the volume of the frame in $\mathrm{m}^{3}$.

\section{Result and Discussion}

The RF loss through vegetation was analysed at $2.4 \mathrm{GHz}$ and for 5 different vegetation thicknesses ( $42 \mathrm{~mm}, 63 \mathrm{~mm}, 105 \mathrm{~mm}, 147 \mathrm{~mm}$ and $195 \mathrm{~mm}$ ). For each thickness, the RF loss in $\mathrm{dB}$ is maximum when the leaves are wet and the RF loss monotonically decreases with the reduction of EWP associated with drying (Figure 3). The calculated value of the RF loss versus EWP (Equation (9)) is also given in the graphs of Figure 3 (red curves).

All graphs exhibit similar gradients (Figure 3(f)) and in all cases the measured RF loss is generally higher than modelled loss irrespective of vegetation thickness. The consistent offset between the measured and modelled values, we believe, is attributable to not adequately accounting for the unbound water in estimating EWP using Equation (21). The method we used to dry the leaves may not have removed the water completely, especially the bound water [23] [24]. When dried to constant weight by heating, vegetation is in an equilibrium state with the drying air [24]. Moreover, the oven dried leaves may then re-absorb water from the air in the period of time between drying and testing. For example, the moisture content of dried and ground alfalfa could be up to $20 \%$ with the normal range of humidity for the time period of testing [24] (Figure 4).

Quantifying the bound water in leaves, on the other hand, is difficult although it can be estimated using a calorimetric methodology [29] [30] [31]. Note this methodology refers to the notion of unbound and bound water as being, respectively, "freezable" and "unfreezable". Assuming they are related, we were unable 


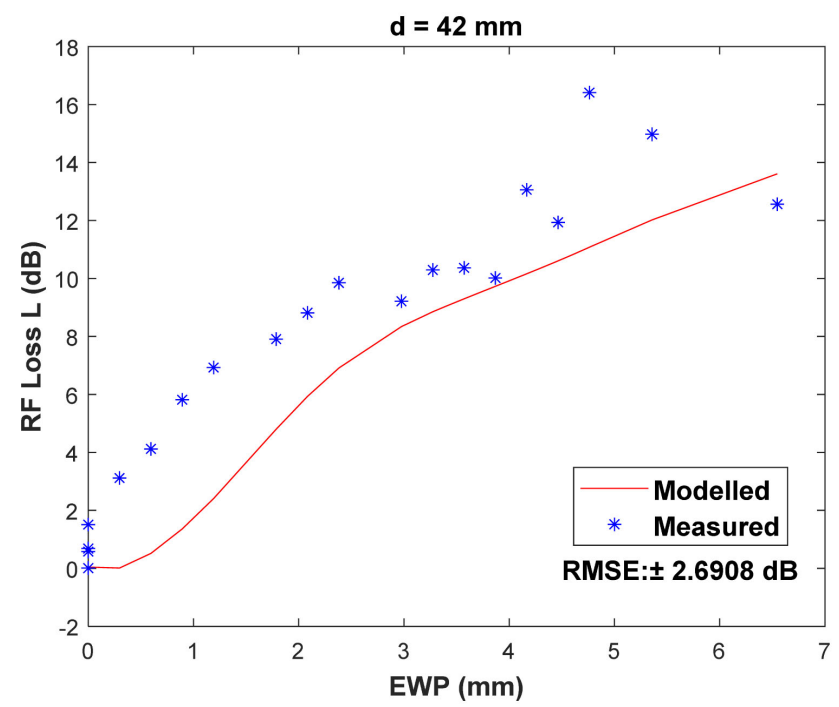

(a)

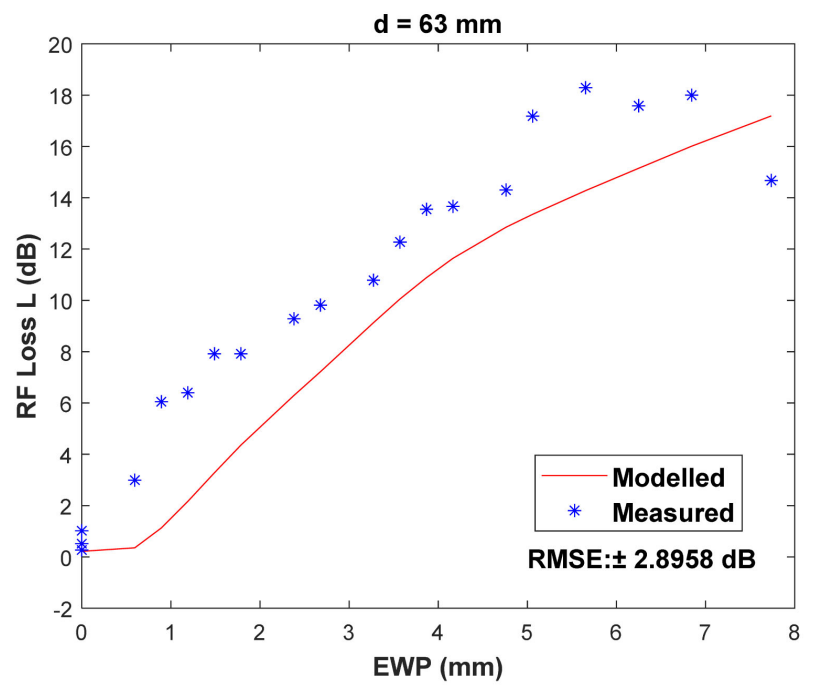

(b)

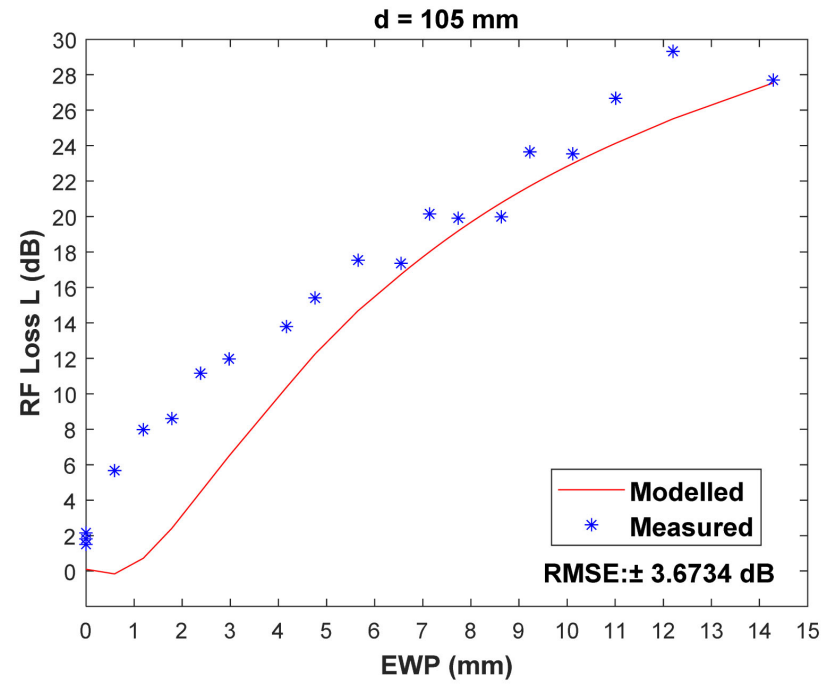

(c) 


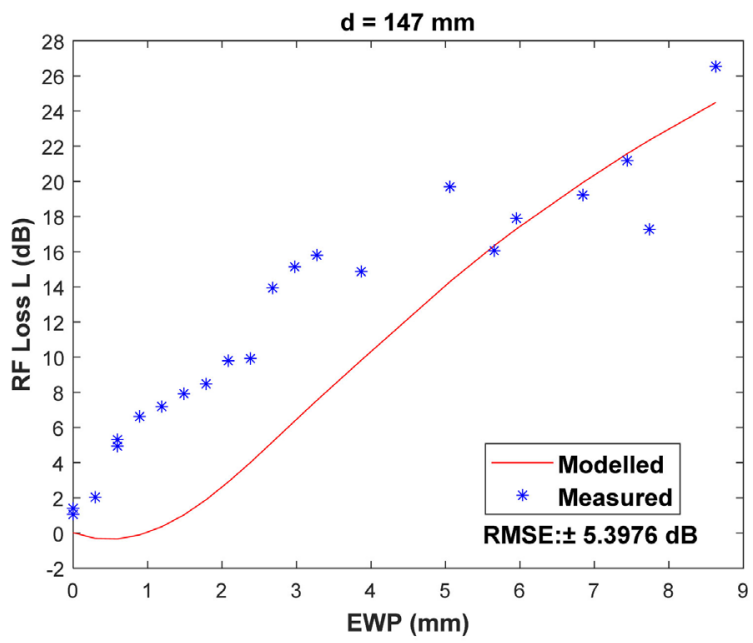

(d)

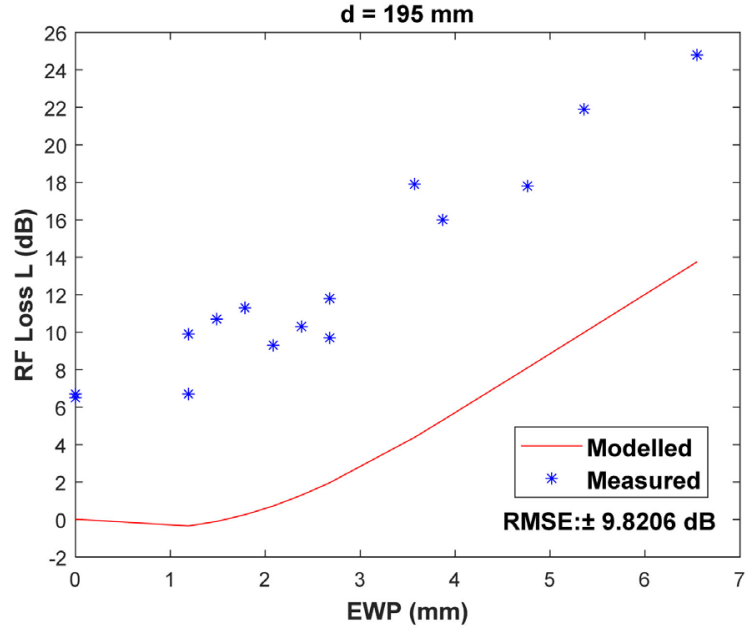

(e)

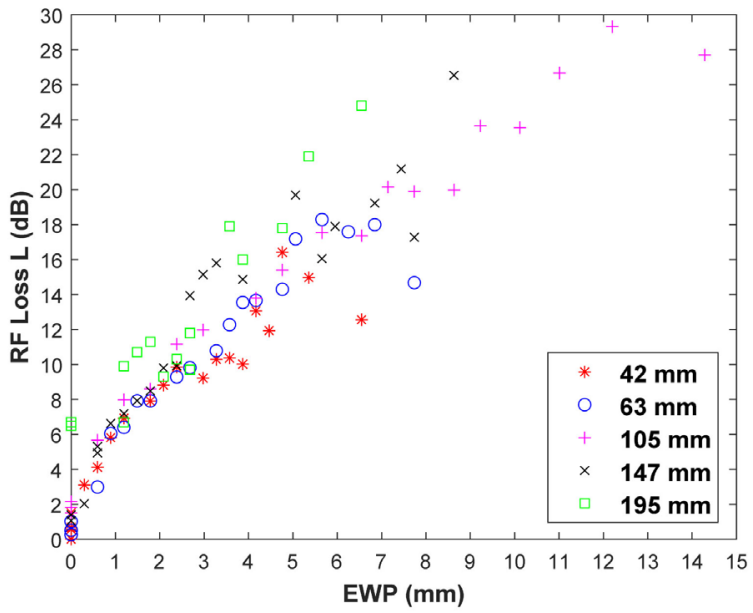

(f)

Figure 3. (a)-(e) Plots of measured and modelled (Equation (9)) RF loss (dB) as functions of EWP (mm) for packed Eucalyptus laevopinea leaves of varying thickness ( $\mathrm{d}=42 \mathrm{~mm}$, $63 \mathrm{~mm}, 105 \mathrm{~mm}, 147 \mathrm{~mm}$ and $195 \mathrm{~mm}$ ), (f) Combined plot of measured RF loss (dB) as a function of EWP for all the five thicknesses. The average RMSE (f) is $\pm 5.6 \mathrm{~dB}$. 


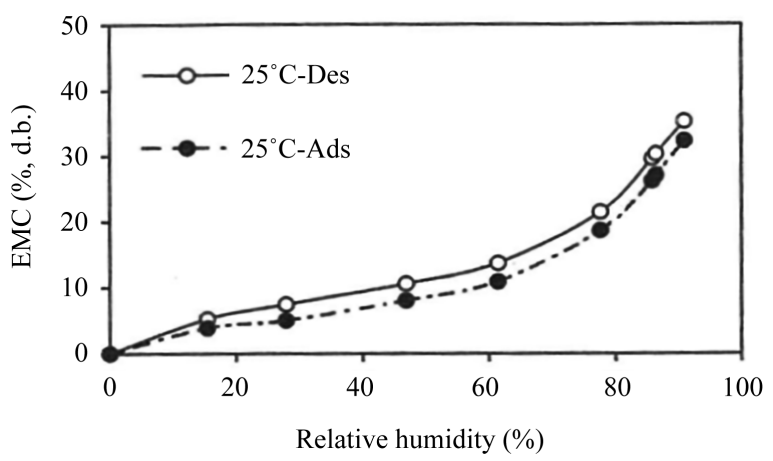

Figure 4. Equilibrium Moisture Content (EMC, \% dry basis) versus Relative Humidity (\%) for ground alfalfa at $25^{\circ} \mathrm{C}$. "Ads" and "Des" stand for adsorption and desorption, respectively [24].

to discern this value for eucalyptus leaves using available literature. However, Whitman [32] provides an insight into at least the possible orders of magnitude of this value on the basis of his work on a range of Prairie grasses in the U.S. during the summer season. Sagebrush, for example, has a bound water content in its leaves ranging from $10 \%-30 \%$ (dry-weight basis) with other grass species exhibiting similar ranges and sometimes higher. In this earlier work, however, the bound water content is measured from freshly-sourced leaves which were not subjected to further desiccation. Here the values would be influenced by external factors such as soil moisture content etc. [32].

An empirical approach available in this work is to identify the value of bound water that would elevate the modelled data values in Figure 3 to the measured values, effectively considering the actual water content of our leaves to be higher (by this additional, bound contribution). We identified this value by finding the minimum total variance between measured and modelled values. The residual water from $1 \%$ to $15 \%$ in $0.1 \%$ increments was evaluated. The best fit between the modelled and the measured values is achieved by assuming that the vegetation contained an additional $6.5 \%$ of water content when dried to constant weight. Residual water of this order of magnitude is plausible when compared against measurements of other leaf types [32]. Of course, what remains unclear is whether or not the "unfreezable" and "freezable" components of water identified by Whitman and others [29] [30] [31] is accessible through the leaf desiccation process in this work (or not) and whether the bound component is a contributor to the RF losses observed in this work.

Nevertheless, and with the new adjustment in the dry weight, the offset between the modelled and measured data collapses, reducing the average RMSE from $\pm 5.6 \mathrm{~dB}$ to $\pm 2.2 \mathrm{~dB}$ (Figure $5(\mathrm{f})$ ). It is notable that the modelled and measured data retain similar gradients; in other words, there appears to be no systematic deviation in the relationship between the amended model and measured values. Despite this encouraging outcome, and in light of the comments above, further work will be undertaken to confirm the value of the bound water components of our leaf samples. 


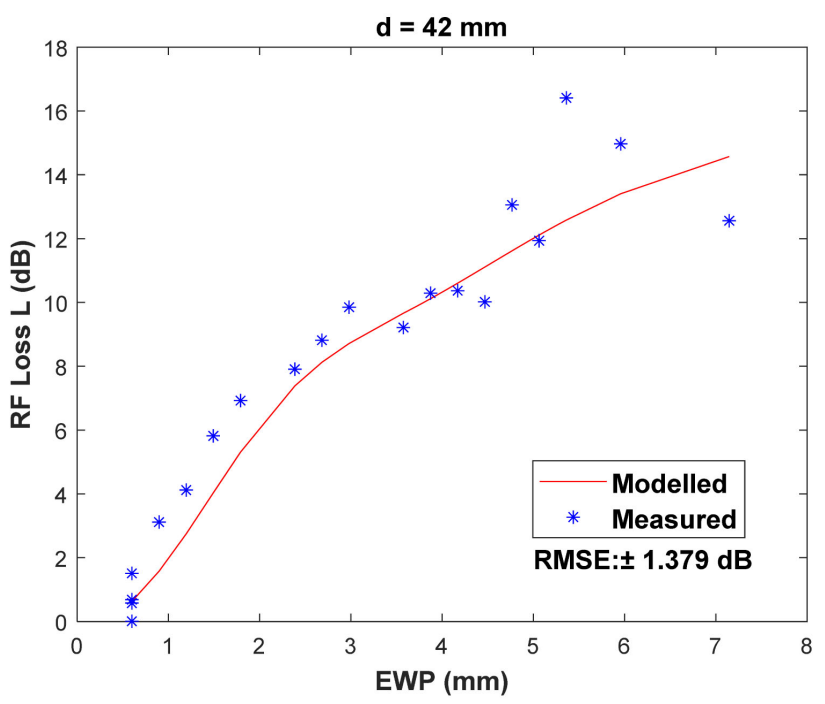

(a)

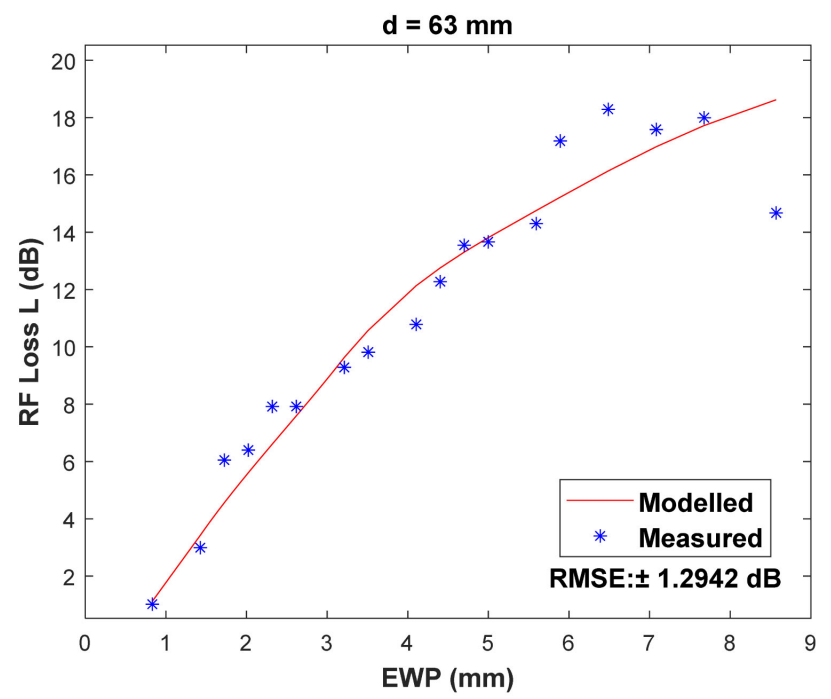

(b)

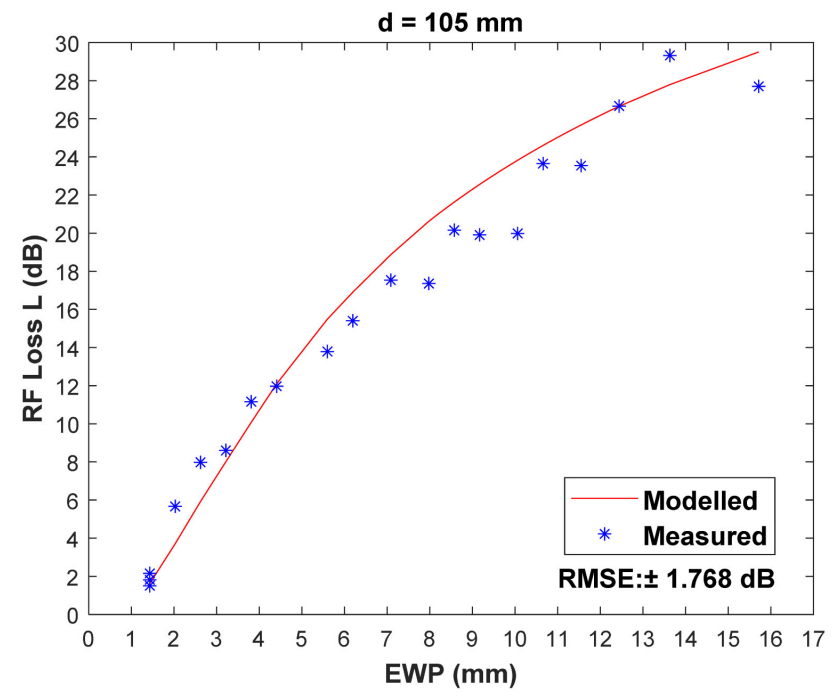

(c) 


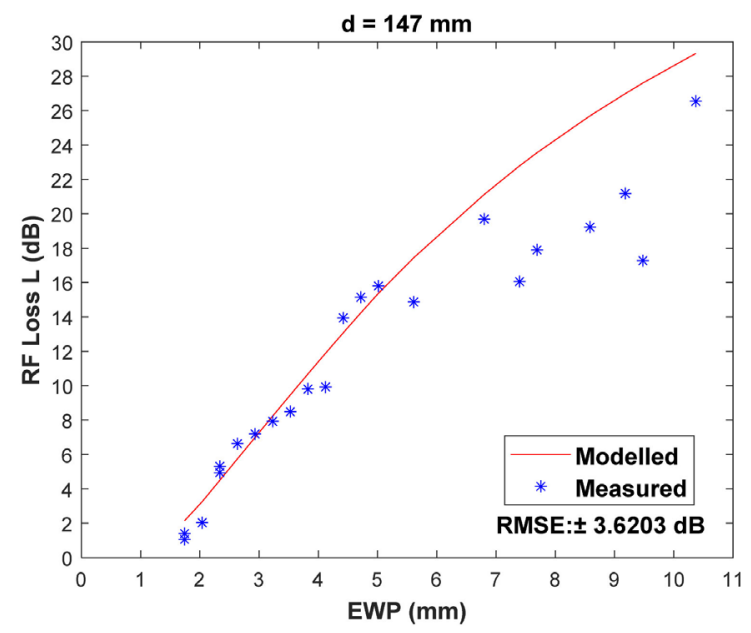

(d)

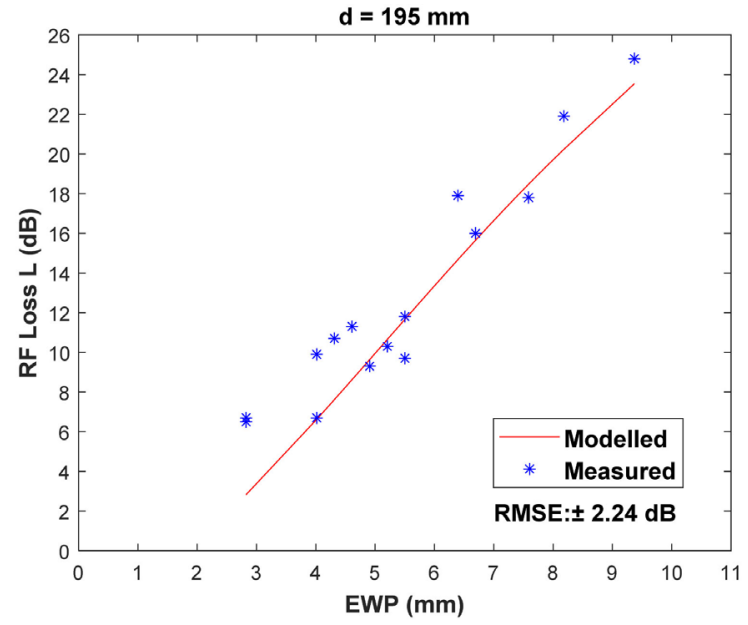

(e)

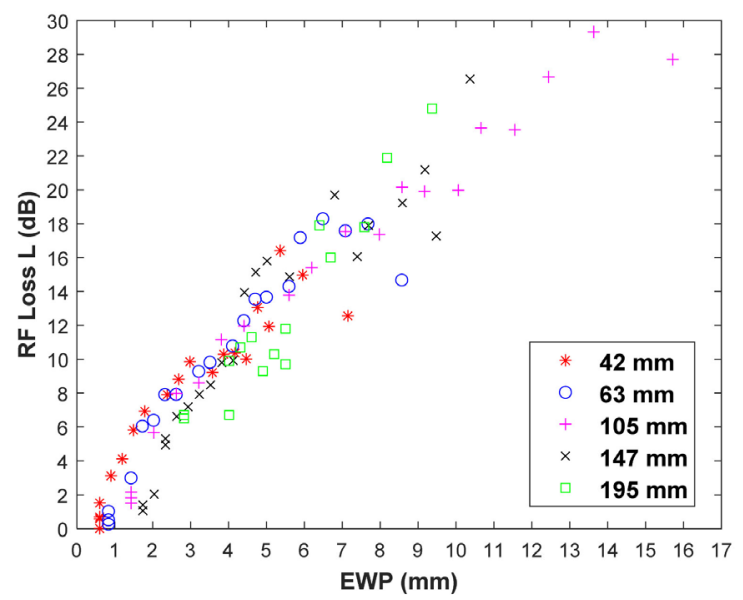

(f)

Figure 5. (a)-(e) Plots of measured and revised-modelled (Equation (9) with additional 6.5\% leaf water content) RF loss as functions of EWP for packed Eucalyptus laevopinea leaves of varying thickness $(\mathrm{d}=42 \mathrm{~mm}, 63 \mathrm{~mm}, 105 \mathrm{~mm}, 147 \mathrm{~mm}$ and $195 \mathrm{~mm})$; (f) Combined plot of measured RF loss (dB) as a function of EWP (with additional 6.5\% leaf water content) for all the five thicknesses. The average RMSE ( $f$ ) is $\pm 2.2 \mathrm{~dB}$. 


\section{Conclusions}

A plane wave model, including an estimation of the water content of leaves, was developed to calculate the RF loss through packed eucalyptus leaves as a function of the effective water path of the assemblages at a frequency of $2.4 \mathrm{GHz}$. There was a positive non-linear relationship between RF loss in $\mathrm{dB}$ and the water content of the leaves when the latter is expressed as effective water path (EWP) in $\mathrm{mm}$. The modelled values versus actual measurements yielded an average RMSE of $\pm 2.2 \mathrm{~dB}$.

Further work is being undertaken by us to relate this mathematical model of radio propagation to empirical measurements of water content and RF loss through whole trees with the view to assessing plant water status for monitoring plant physiology.

\section{Acknowledgements}

The first author acknowledges receipt of a Tuition Fee-Wavier Scholarship from the University of New England. DWL acknowledges the support of Food Agility CRC Ltd, funded under the Commonwealth Government CRC Program. The CRC Program supports industry-led collaborations between industry, researchers, and the community. All authors gratefully acknowledge the contribution of Derek Schneider and Patrick Littlefield from UNE for their help in setting up the experiment, and Professor Jeremy Bruhl from UNE for helping us identify the eucalyptus species used for the experiment.

\section{Conflicts of Interest}

The authors declare no conflicts of interest regarding the publication of this paper.

\section{References}

[1] LaGrone, A.H. (1960) Forecasting Television Service Fields. Proceedings of the IRE, 48, 1009-1015. https://doi.org/10.1109/JRPROC.1960.287501

[2] Balachander, D., Rao, T.R. and Mahesh, G. (2013) RF Propagation Experiments in Agricultural Fields and Gardens for Wireless Sensor Communications. Progress in Electromagnetics Research C, 39, 103-118. https://doi.org/10.2528/PIERC13030710

[3] Hristos, T.A., Stavros, V., Theodoros, F., Christian, R., Loukas, P., Manuela, Z. and Jana, K. (2014) A Computational Model for Path Loss in Wireless Sensor Networks in Orchard Environments. Sensors, 14, 5118-5135. https://doi.org/10.3390/s140305118

[4] Weissberger, M.A. (1982) An Initial Critical Summary of Models for Predicting the Attenuation of Radio Waves by Trees. https://doi.org/10.21236/ADA118343

[5] Seville, A. and Craig, K.H. (1995) Semi-Empirical Model for Millimetre-Wave Vegetation Attenuation Rates. Electronics Letters, 31, 1507-1508. https://doi.org/10.1049/el:19951000

[6] Al-Nuaimi, M.O. and Stephens, R.B.L. (1998) Measurements and Prediction Model Optimisation for Signal Attenuation in Vegetation Media at Centimetre Wave Frequencies. IEE Proceedings-Microwaves, Antennas and Propagation, 145, 201-206. 
https://doi.org/10.1049/ip-map:19981883

[7] Adegoke, A.S. (2014) Measurement of Propagation Loss in Trees at SHF Frequencies. Ph.D. Thesis, University of Leicester, Leicester.

[8] Guo, X.-M. and Zhao, C. (2014) Propagation Model for 2.4 GHz Wireless Sensor Network in Four-year-old Young Apple Orchard. International Journal of Agricultural and Biological Engineering, 7, 47-53.

[9] Rogers, N.C., Seville, A., Richter, J., Ndzi, D., Savage, N., Caldeirinha, R., Shukla, A., Al-Nuaimi, M., Craig, K. and Vilar, E. (2002) A Generic Model of 1-60 GHz Radio Propagation through Vegetation-Final Report. Radio Agency, UK.

[10] Peñuelas, J., Filella, I., Biel, C., Serrano, L. and Save, R. (1993) The Reflectance at the 950-970 nm Region as an Indicator of Plant Water Status. International journal of Remote Sensing, 14, 1887-1905. https://doi.org/10.1080/01431169308954010

[11] Peñuelas, J., Filella, I., Serrano, L. and Save, R. (1996) Cell Wall Elasticity and Water Index (R970 nm/R900 nm) in Wheat under Different Nitrogen Availabilities. International Journal of Remote Sensing, 17, 373-382.

https://doi.org/10.1080/01431169608949012

[12] Datt, B. (1999) Remote Sensing of Water Content in Eucalyptus Leaves. Australian Journal of Botany, 47, 909-923. https://doi.org/10.1071/BT98042

[13] Jiang, S. and Georgakopoulos, S. (2011) Electromagnetic Wave Propagation into Fresh Water. Journal of Electromagnetic Analysis and Applications, 3, 261-266. https://doi.org/10.4236/jemaa.2011.37042

[14] Da Silva, M.J. (2008) Impedance Sensors for Fast Multiphase Flow Measurement and Imaging. Ph.D. Thesis, Technische Universität Dresden, Dresden.

[15] Lunkenheimer, P., Emmert, S., Gulich, R., Köhler, M., Wolf, M., Schwab, M. and Loidl, A. (2017) Electromagnetic-Radiation Absorption by Water. Physical Review E, 96, 062607. https://doi.org/10.1103/PhysRevE.96.062607

[16] Le Vine, D.M. and Karam, M.A. (1996) Dependence of Attenuation in a Vegetation Canopy on Frequency and Plant Water Content. IEEE Transactions on Geoscience and Remote Sensing, 34, 1090-1096. https://doi.org/10.1109/36.536525

[17] Nakajima, I., Ohyama, F., Juzoji, H. and Ta, M. (2019) Developing a Scanner for Assessing Foliage Moisture. Journal of Multimedia Information System, 6, 155-164. https://doi.org/10.33851/JMIS.2019.6.3.155

[18] Schubert, F.M., Fleury, B.H. and Prieto-Cerdeira, R. (2009) Propagation Model for Wave Scattering Effects Caused by Trees. COST Action IC0802 2nd Management Committee Meeting, Toulouse, France, 4-6 November 2009.

[19] Caldeirinha, R.F.S., Morgadinho, S., Frazão, L., Cuiñas, I., Sanchéz, M. and Al-Nuaimi, M. (2006) Wind Incidence Effects on Channel Dynamics in Vegetation Media at $40 \mathrm{GHz}$. 2006 IEEE International Symposium on Geoscience and Remote Sensing, Denver, 31 July-4 August 2006, 1032-1035. https://doi.org/10.1109/IGARSS.2006.266

[20] Benzair, B. (1993) Characterisation of Radio Wave Propagation Inside Building and Through Vegetation. Ph.D. Thesis, University of Bradford, Bradford.

[21] Al Salameh, M.S.H. (2019) Predicting Leaf State Effects on Radiowaves Based on Propagation Loss Measurements. MATEC Web of Conference, EDP Sciences, 12, 02005. https://doi.org/10.1051/matecconf/201929202005

[22] Shmulsky, R. and Jones, P.D. (2011) Wood and Water. In: Forest Products and Wood Science: An Introduction, 6th Edition, John Wiley \& Sons, Hoboken, 141-174. https://doi.org/10.1002/9780470960035.ch7 
[23] Hasted, J.B. (1973) Aqueous Dielectrics. Chapman and Hall, London.

[24] Yang, W. and Siebenmorgen, T. (2003) Drying Theory. In: Encyclopedia of Agricultural, Food, and Biological Engineering, Marcel Dekker, New York, 231-241.

[25] Ulaby, F.T. and El-Rayes, M.A. (1987) Microwave Dielectric Spectrum of Vegetation-Part II: Dual-Dispersion Model. IEEE Transactions on Geoscience and Remote Sensing, GE-25, 550-557. https://doi.org/10.1109/TGRS.1987.289833

[26] Balanis, C.A. (2012) Advanced Engineering Electromagnetics. John Wiley \& Sons, Hoboken.

[27] Ulaby, F.T., Moore, R.K. and Fung, A.K. (1986) Microwave Remote Sensing: Active and Passive: Volume 3-From Theory to Applications. Artech House, Norwood, US.

[28] El-Rayes, M.A. (1987) The Measurement and Modeling of the Dielectric Behavior of Vegetation Materials in the Microwave Region (0.5-20.4 GHZ). Ph.D. Thesis, University of Kansas, Lawrence.

[29] Robinson, W. (1931) Free and Bound Water Determinations by the Heat of Fusion of Ice Method. Journal of Biological Chemistry, 92, 699-709. https://doi.org/10.1016/S0021-9258(17)32613-3

[30] Sayre, J. (1932) Methods of Determining Bound Water in Plant Tissue. Journal of Agricultural Research, 44, 669-688.

[31] Stark, A. (1932) An Apparatus and Method for Determining Bound Water in Plant Tissue. Proceedings of the American Society for Horticultural Science, 29, 384-388.

[32] Whitman, W.C. (1941) Seasonal Changes in Bound Water Content of Some Prairie Grasses. Botanical Gazette, 103, 38-63. https://doi.org/10.1086/335024 